\title{
Contributors to the 2020 IMIA Yearbook of Medical Informatics
}

\author{
Editors: \\ Kate Fultz Hollis \\ Oregon Health \& Science University \\ Portland, OR, USA \\ fultzhol@ohsu.edu

Brigitte Séroussi
Sorbonne University \& LIMIICS UMR_S 1142
Tenon Hospital, AP-HP
Paris, France
brigitte.seroussi@aaphp.fr
Lina F. Soualmia
Normandie University, UNIIROUEN, LITIS
Rouen, France
Lina.Soualmia@chu-roven.fr \\ Editorial Assistant: \\ Martina Hutter \\ Heidelberg University Hospital - ZIM \\ Heidelberg, Germany \\ yearbook@imia-services.org
}

Best Papers Editor:

Adrien Ugon

ESIEE Paris \& LPF UMR 7606

adrien.ugon@esiee.fr

Advisory Board:

Reinhold Houx

Peter L. Reichertz Institute for Medical Informatics

of TU Braunschweig and Hannover

Medical School

Braunschweig, Germany

reinhold.haux@plri.de

Victor Maojo

Universidad Politécnica de Madrid

Departamento de Inteligencia Arificicial

Facultad de Informatica

Madrid, Spain

vmaoj@@fi.upm.es

George Mihalos

University of Medicine and Pharmacy

Dept. of Medical Informatics

Timisoara, Romania

mihalas@EFMl.info

\begin{tabular}{|c|}
\hline $\begin{array}{l}\text { Section Editors: } \\
\text { Christian Baumgartner } \\
\text { Graz University of Technology } \\
\text { Graz, Austria } \\
\text { christian.baumgartner@tugraz.at }\end{array}$ \\
\hline $\begin{array}{l}\text { Eta S. Berner } \\
\text { Department of Health Services Administration } \\
\text { University of Alabama at Birmingham } \\
\text { Bimingham, AL, USA } \\
\text { eberner@uab.edu }\end{array}$ \\
\hline $\begin{array}{l}\text { Meryl Bloomrosen } \\
\text { Premier healthcare alliance } \\
\text { Washington, DC } \\
\text { meryl_bloomrosen@premierinc.com }\end{array}$ \\
\hline $\begin{array}{l}\text { Jacques Bouaud } \\
\text { DRCI/AP-HP \& LIMICS UMR_S } 1142 \\
\text { Paris, France } \\
\text { jacques.bouaud@aphp.fr }\end{array}$ \\
\hline $\begin{array}{l}\text { Jean Charlet } \\
\text { DRCI/AP-HP \& LIMICS UMR_S } 1142 \\
\text { Paris, France } \\
\text { jean.charlet@upmc.fr }\end{array}$ \\
\hline $\begin{array}{l}\text { Sebastien Cossin } \\
\text { Bordeaux University \& Inserm UMR } 1219 \\
\text { Bordeaux, France } \\
\text { sebastien.cossin@u-bordeaux.fr }\end{array}$ \\
\hline $\begin{array}{l}\text { Christel Daniel } \\
\text { WIND-DSI/AP-HP \& LIMISC UMR_S } 1142 \\
\text { Paris, France } \\
\text { christel.daniel@aphp.fr }\end{array}$ \\
\hline $\begin{array}{l}\text { Thomas M. Deserno } \\
\text { Peter L. Reichertz Institute for Medical Informatic } \\
\text { of TU Braunschweig and Hannover } \\
\text { Medical School } \\
\text { Braunschweig, Germany } \\
\text { thomas.deserno@plri.de }\end{array}$ \\
\hline $\begin{array}{l}\text { Ferdinand Dhombres } \\
\text { Sorbonne University \& LMIICS UMR_S } 1142 \\
\text { Trousseau Hospital, AP-HP } \\
\text { Paris, France } \\
\text { ferdinand.dhombres@aphp.fr }\end{array}$ \\
\hline $\begin{array}{l}\text { Catherine Duclos } \\
\text { Sorbonne Paris Nord University } \\
\text { \& LMIICS UMR S } 1142 \\
\text { Ancienne Hospital, AP-HP } \\
\text { Bobigny, France } \\
\text { catherine.duclos@aphp.fr }\end{array}$ \\
\hline $\begin{array}{l}\text { Natalia Grabar } \\
\text { cNRS, Univ. Lille, UMR } 8163 \\
\text { STL- Savoirs Textes Langage } \\
\text { lille, France } \\
\text { natalia.grabar@univ-lille.fr }\end{array}$ \\
\hline
\end{tabular}

Cyril Grouin

LIMSI, CNRS, Université Paris Saday

Orsay, France

cyril.grouin@limsi.fr

Werner Hackl

UMIT - Private University for Health Sciences,

Medical Informatics and Technology

Hall in Tirol, Austria

werner.hack@umit.ot

Alexander Hoerbst

MCl - The Entrepreneurial School

Innsbruck, Austria

alexander.hoerbst@mci.edu

William Hsu

University of California Los Angeles

Los Angeles, CA, USA

whsu@mednet.ucla.edu

Dipak Kalra

The University of Gent

Gent, Belgium

dipak.kalra@i-hd.eu

Annie Y. S. Lau

Centre for Health Informatics

Australian Institute of Health Innovation

Macquarie University, Australia

annie.lau@mq.edu.au

Debra Patt

Texas Oncology

Dell Medical School

at The University of Texas at Austin

Austin, Texas, USA

debra.pati@usoncology.com

Sylvia Pelayo

Lille University \& CHU Lille

Lille, France

sylvia.pelayo@univ-lille.fr

Carolyn Petersen

Division of Biomedical Statistics and Informatics

Mayo Clinic

Rochester, Minnesota, USA

petersen.carolyn@mayo.edu

Bastien Rance

HEGP/AP-HP \& Université Paris Descartes

INSERM UMRS 1138

Paris, France

bastien.rance@uphp.fr

Yalini Senathirajah

University of Pittsburgh School of Medicine

Pittsburgh, USA

yalini@pitt.edu
Malika Smaïl-Tabonne

Loria UMR 7503

Université de Lorraine

Nancy, France

malika.smail@loria.fr

Pascal Staccini

IRIS Department, Lab RETINES

Faculté de Médecine, Université Côte d'Azur

Nice, France

pascal.staccini@univ-cotedazur.fr

Vignesh Subbian

College of Engineering

The University of Arizona

Tucson, Arizona, USA

vsubbian@arizona.edu

Rodolphe Thiébaut

Inserm U1219 \& Inria SISTM

Bordeaux University

Bordeaux, France

rodolphe.thiebaut@u-bordeaux.fr

Jeremy L. Warner

Vanderbilt University

Nashville, TN, USA

jeremy.warner@vumc.org

\section{Regional Editors:}

Vaijra Dissanayake

APAMI

president@apami.org

Ghislain B. Kovematchoua Tchuitchev HELINA

kouematchoua@helina-online.org

Rivad Al Shammari

MENAHIA

riyadalshammari@gmail.com

Karen Greenwood

AMIA

Bethesda, MD, USA

karen@amia.org

Andre Kushniruk

Digital Health Canada: Canada's Health

Informatics Association

Toronto, ON, Canada

andrek@uvic.ca

Christian Lovis

EFMI

christian.lovis@hcuge.ch

IMIA Executive Director:

Elaine Huesing

imia@imia-services.org 